\title{
Experience Profiling of Fluorescence-Guided Surgery I: Gliomas
}

\author{
So Young Ji', Jin Wook Kim ${ }^{1,2}$, Chul-Kee Park ${ }^{1,2}$ \\ ${ }^{1}$ Department of Neurosurgery, Seoul National University Hospital, Seoul, Korea \\ ${ }^{2}$ Department of Neurosurgery, Seoul National University College of Medicine, Seoul, Korea
}

\author{
Received June 22, 2019 \\ Revised July 23, 2019 \\ Accepted August 6, 2019

\section{Correspondence} \\ Chul-Kee Park \\ Department of Neurosurgery, \\ Seoul National University Hospital, \\ Seoul National University \\ College of Medicine, \\ 101 Daehak-ro, Jongno-gu, \\ Seoul 03080, Korea \\ Tel: +82-2-2072-0347 \\ Fax: +82-504-154-4633 \\ E-mail: nschpark@snu.ac.kr
}

\begin{abstract}
Background Numerous studies reported a usefulness of 5-aminolevulinic acid (5-ALA) fluorescence-guided surgery (FGS) in high grade gliomas. However, fluorescence patterns and intensities are variable among gliomas. In this study, we report our extensive experience with FGS in various gliomas, focusing on epidemiological data of fluorescence patterns.
\end{abstract}

Methods A total of 827 histologically proven glioma patients out of 900 brain tumor patients who had undergone FGS using 5-ALA during the period of 8.5 years between July 2010 and January 2019 were analyzed. Indications of FGS in glioma surgery are evidence for possible high-grade foci in putative gliomas in preoperative MRI.

Results Among the 827 gliomas, the number of cases corresponding to 2016 World Health Organization (WHO) grade IV, III, II, and I are 528 (58.7\%), 193 (21.4\%), 87 (9.7\%) and 19 (2.1\%), respectively. In terms of fluorescence rate, grade IV gliomas showed positive fluorescence in $95.4 \%$ of cases including strong intensity in $85.6 \%$. Grade III gliomas showed fluorescence in about half of cases (55.0\%), but $45.0 \%$ of the cases showed no fluorescence at all. Anaplastic oligodendroglioma had a higher positive rate (63.9\%) than anaplastic astrocytoma (46.2\%). Both grade II and I gliomas still showed positive fluorescence in about one-fourth of cases (24.1\% and $26.3 \%$ respectively). Among them ependymoma and pilocytic astrocytoma were fluorescence-prone tumors.

Conclusion This epidemiological data of 5-ALA fluorescence in various grades of glioma provides a basic reference to the clinical application of FGS with 5-ALA in glioma surgery.

Key Words Fluorescence; Gliomas; Glioblastoma; Surgery.

\section{INTRODUCTION}

Complete removal of intracranial gliomas remains a challenge for many neurosurgeons. Maximum safe resection is important not only for low-grade gliomas but also for highgrade gliomas that improve progression-free survival and overall survival as well as the efficacy of adjuvant therapy [16]. However, the invasive and vague tumor margins of glioma make it difficult to achieve complete resection. Over the years, efforts to develop new tools for visualization of malignant brain tumors have come to fruition. One of them is fluorescence-guided surgery (FGS) using 5-aminolevulinic

This is an Open Access article distributed under the terms of the Creative Commons Attribution Non-Commercial License (https://creativecommons.org/licenses/by-nc/4.0) which permits unrestricted non-commercial use, distribution, and reproduction in any medium, provided the original work is properly cited.

Copyright $\odot 2019$ The Korean Brain Tumor Society, The Korean Society for NeuroOncology, and The Korean Society for Pediatric Neuro-Oncology acid (5-ALA), which has become widely used in glioma surgery worldwide [7-10]. 5-ALA causes transient accumulation of fluorescent protoporphyrin IX (PpIX) in malignant cells through alteration of heme biosynthetic pathway $[10,11]$. Cumulative PpIX enables intraoperative visualization of malignant tissue under a microscope with a long pass filter [12].

Numerous studies reported safety and usefulness of 5-ALA FGS in glioblastomas [13]. Although the efficacy of 5-ALA FGS has been reported in other types of gliomas, the fluorescence pattern is not as uniform as glioblastoma [1416]. In low grade gliomas, 5-ALA fluorescence is absent in most cases but not all cases [17]. In addition, there is intratumoral heterogeneity of the fluorescence pattern [18]. In this study, we report our extensive experience with FGS in various gliomas, focusing on the epidemiological data of fluorescence patterns and their impact on the extent of resection. 


\section{MATERIALS AND METHODS}

A total of 827 histologically proven glioma patients out of 900 brain tumor patients who had undergone FGS using 5 -ALA during the period of 8.5 years between July 2010 and January 2019 were analyzed. The 900 FGS for brain tumor is $15.8 \%$ of all adult brain tumor surgeries $(n=5,682)$ performed in Seoul National University Hospital, and FGS was applied to $50.8 \%$ of all glioma surgeries $(n=1,629)$ during the same period.

The indication for FGS in glioma surgery was that preoperative MRI has the potential for high-grade glioma foci such as diffusion restriction, increased cerebral blood volume, and enhancement. On the other hand, FGS was not considered in the absence of radiological evidence such as high grade lesions, the need for awake-surgery (owing to facial photosensitivity in operation theater atmosphere), heart problems with complications, and poor medical condition such as liver or kidney failures. We did not used 5-ALA for the stereotactic or navigational biopsies even if the impression of tumor was high grade glioma. The patients' age ranged from 16 to 84 years (mean 50 years). This study was approved for retrospective analysis by Institutional Review Board of Seoul National University Hospital (IRB No: 1905-093-1034).

All the patients were administered $20 \mathrm{mg} / \mathrm{kg}$ of 5-ALA (Gliolan $^{\circledR}$; Medac, Wedel, Germany) mixed with $50 \mathrm{~mL}$ of water 3 to 4 hours before the induction of general anesthesia. Fluorescence-guided surgeries were done with a Leica M720 OH5 microscope (Leica, Wetzlar, Germany) equipped with an FL400 Fluorescence module (Leica, Wetzlar, Germany) or a Zeiss Pentero equipped with a fluorescent $400 \mathrm{~nm}$ UV light and filters (Zeiss, Oberkochen, Germany). Regarding to the fluorescence intensity of the tumor, optical judgement was made during the surgery and recorded classifying red for "strong" intensity, pink for "weak" intensity, and blue for "no" fluorescence. Post hoc evaluation was performed using operative pictures and videos for those ambiguous cases.

In all patients, preoperative MRI was performed the day before surgery, and postoperative MRI was performed within 48 hours after surgery to compare the extent of resection. Complete resection was judged if there are no measurable lesions by postoperative MRI. A measurable lesion is defined as a contrast-enhancing lesion with maximal diameter and second perpendicular measurement at least $10 \mathrm{~mm}$ in size. Non-enhancing flair lesions on postoperative MRI were considered residual tumors if the surgeon's opinion indicated that the resection was incomplete.

\section{RESULT}

Distribution of histological diagnosis of 900 brain tumor patients including 827 gliomas who had undergone FGS are summarized in Fig. 1A. Among the 827 gliomas, the number of cases corresponding to 2016 World Health Organization (WHO) grade IV, III, II, and I were 528 (58.7\%), 193 (21.4\%), 87 (9.7\%) and 19 (2.1\%), respectively. The distribution of detailed histological subtypes is described in Table 1.

The fluorescence intensity showed differences in grades (Fig. 1B). Grade IV gliomas showed positive fluorescence in $95.4 \%$ of cases including strong intensity in $85.6 \%$. Grade III gliomas showed fluorescence in about half (55\%) of cases, but $45 \%$ of cases showed no fluorescence. Interestingly, grade II and I gliomas likewise showed positive fluorescence in
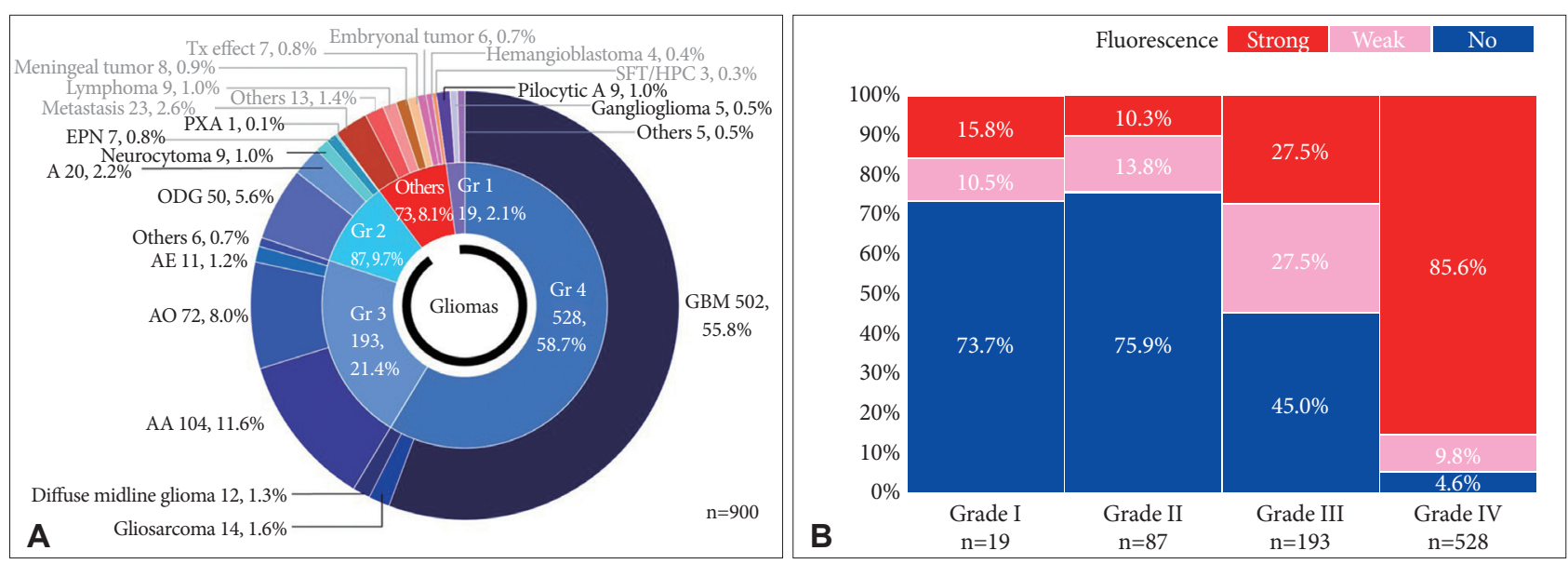

Fig. 1. A: Distribution of histological diagnosis of 900 fluorescence-guided surgery cases including 827 gliomas. Experience of other 73 non-glioma cases (gray text) are described in the other paper in this issue (Experience profiling of fluorescence-guided surgery II: non-glioma pathologies). Numbers in diagram indicate number of cases. B: Distribution of fluorescence intensity of 827 gliomas classified by World Health Organization (WHO) grade. GBM, glioblastoma; AA, anaplastic astrocytoma; AO, anaplastic oligodendroglioma; AE, anaplastic ependymoma; ODG, oligodendroglioma; A, astrocytoma; EPN, ependymoma; PXA, pleomorphic xanthoastrocytoma; Gr, grade. Tx: treatment, SFT: solitary fibrous tumor, HPC: hemangiopericytoma. 
Table 1. Characteristics of the WHO grade of gliomas and their histological subtypes

\begin{tabular}{lc}
\hline \multicolumn{1}{c}{ WHO grade } & Number of cases (\%) \\
\hline Grade IV & $528(58.7)$ \\
Glioblastoma & $502(55.8)$ \\
Gliosarcoma & $14(1.6)$ \\
Diffuse midline gliom K27M-mutant & $12(1.3)$ \\
Grade III & $193(21.4)$ \\
Anaplastic astrocytoma/oligoastrocytoma & $104(11.6)$ \\
Anaplastic oligodendroglioma & $72(8.0)$ \\
Anaplastic ependymoma & $11(1.2)$ \\
Gliomatosis cerebri & $1(0.1)$ \\
Glioneuronal tumor grade III & $1(0.1)$ \\
Anaplastic ganglioglioma & $4(0.5)$ \\
Grade II & $87(9.7)$ \\
Oligodendroglioma & $50(5.2)$ \\
Diffuse astrocytoma/Oligoastrocytoma & $20(2.2)$ \\
Ependymoma & $7(0.8)$ \\
Central neurocytoma/ & $9(1.0)$ \\
Extra ventricular neurocytoma & \\
Pleomorphic xanthoastrocytoma & $1(0.1)$ \\
Grade I & $19(2.1)$ \\
Pilocytic astrocytoma & $9(1.0)$ \\
Ganglioglioma & $5(0.6)$ \\
Dysembryoplastic neuroepithelial tumor & $3(0.4)$ \\
Choroid plexus papilloma & $1(0.1)$ \\
Papillary glioneuronal tumor & $1(0.1)$ \\
Total & 827 \\
\hline &
\end{tabular}

about one quarter of cases (24.1\% and $26.3 \%)$.

\section{Grade IV gliomas}

Of the 502 glioblastomas, $96 \%(n=482)$ cases were positive for fluorescence, and most showed strong red fluorescence ( $\mathrm{n}=434,86.5 \%)$ (Fig. 2A). There were 20 cases (3.9\%) of glioblastoma without any fluorescence. These 20 fluorescence negative glioblastomas did not exhibit any common radiological characteristics that could predict intraoperative 5-ALA fluorescence. Five cases had no enhancement on pre-operative MRI, but the other 15 cases showed strong enhancement (Fig. 2B). In some cases, the assumptions that limit the visibility of fluorescence include overtime (failure to match fluorescence time window), overweight of patient (dose insufficiency), hyper-vascularity (poor visibility of operation field), and extensive necrosis (Fig 2B). Complete resection could be achieved in $89.6 \%$ of cases showing fluorescence positive (432/482 patients), but only $75.0 \%$ of fluorescence negative cases (15/20 cases) succeeded in complete resection (Fig. 2A). There was a significant difference in the complete resection rate according to fluorescence positive $(p=0.042)$. Of the 55 cases that could not be completely removed, 50 were fluores- cence positive. The reasons for incomplete excision despite positive fluorescence most often include eloquent location and extensive nature of tumor $(n=37)$. Other miscellaneous reasons are multifocality of tumor, hypervascularity, inadequate surgical window, and critical vessel attachment.

A total of 14 gliosarcomas were registered in this series. All gliosarcoma cases, including 12 (85.7\%) strong fluorescence and 2 (14.3\%) weak fluorescence showed positive fluorescence. All cases were successfully resected completely, except for one case where the surgical window had a problem.

Diffuse midline glioma, recently raised a relatively novel disease entity, are identified in 12 cases. Fluorescence positivity was $66.7 \%(n=8)$, including 6 strong and 2 weak (Fig. 2A). Of these eight fluorescence positive cases, two cases were incompletely resected due to extensive and eloquent location (Fig. 2A).

\section{Grade III gliomas}

Among the 193 grade III gliomas, anaplastic astrocytoma covered $53.8 \%(\mathrm{n}=104)$ followed by anaplastic oligodendroglioma $(37.3 \%, n=72)$, anaplastic ependymoma $(5.7 \%, n=11)$, anaplastic ganglioglioma $(2.1 \%, n=4)$, and other 2 miscellaneous tumors. Anaplastic astrocytoma showed positive fluorescence in $46.2 \%$ of cases $(n=48)$ including strong fluorescence in $26.0 \%(n=27)$, and weak fluorescence in 20.2\% ( $n=21)$ (Fig. 3). More than half of anaplastic astrocytoma cases showed no fluorescent $(53.8 \%, \mathrm{n}=56)$. In terms of complete resection rate, there was insignificant difference $(p=0.695)$ between anaplastic astrocytomas with fluorescence $(83.3 \%)$ and without fluorescence (80.4\%). Most of the reasons for incomplete excision in grade III astrocytomas, with or without fluorescence, have been the involvement of extensive nature and/or eloquent areas.

Compared to anaplastic astrocytoma (46.2\%), there were more fluorescence cases positive for anaplastic oligodendroglioma $(63.9 \%, n=46)$. Among them, 26.4\% $(n=19)$ anaplastic oligodendroglioma showed strong fluorescence and $37.5 \%(n=27)$ showed weak fluorescence (Fig. 3). Twenty-six cases (36.1\%) of anaplastic oligodendrogliomas still showed no fluorescence. Similar to anaplastic astrocytomas, the complete resection rate of anaplastic oligodendroglioma was not significantly different by fluorescence (91.3\% without fluorescence, vs. $88.5 \%$ without fluorescence). The reason for incomplete resection is almost always due to the extensive nature of the tumor.

Of the 11 cases of anaplastic ependymoma, there was strong fluorescence in 5 cases, weak fluorescence in 3 cases, and the other 3 cases showed no fluorescence (Fig. 3). All undifferentiated ependymomas, except for one broad tumor without fluorescence, could be completely resected. There were 4 cases of anaplastic gangliogliomas, 2 of which showed strong fluorescence, while the other 2 cases showed no fluorescence (Fig. 3). 

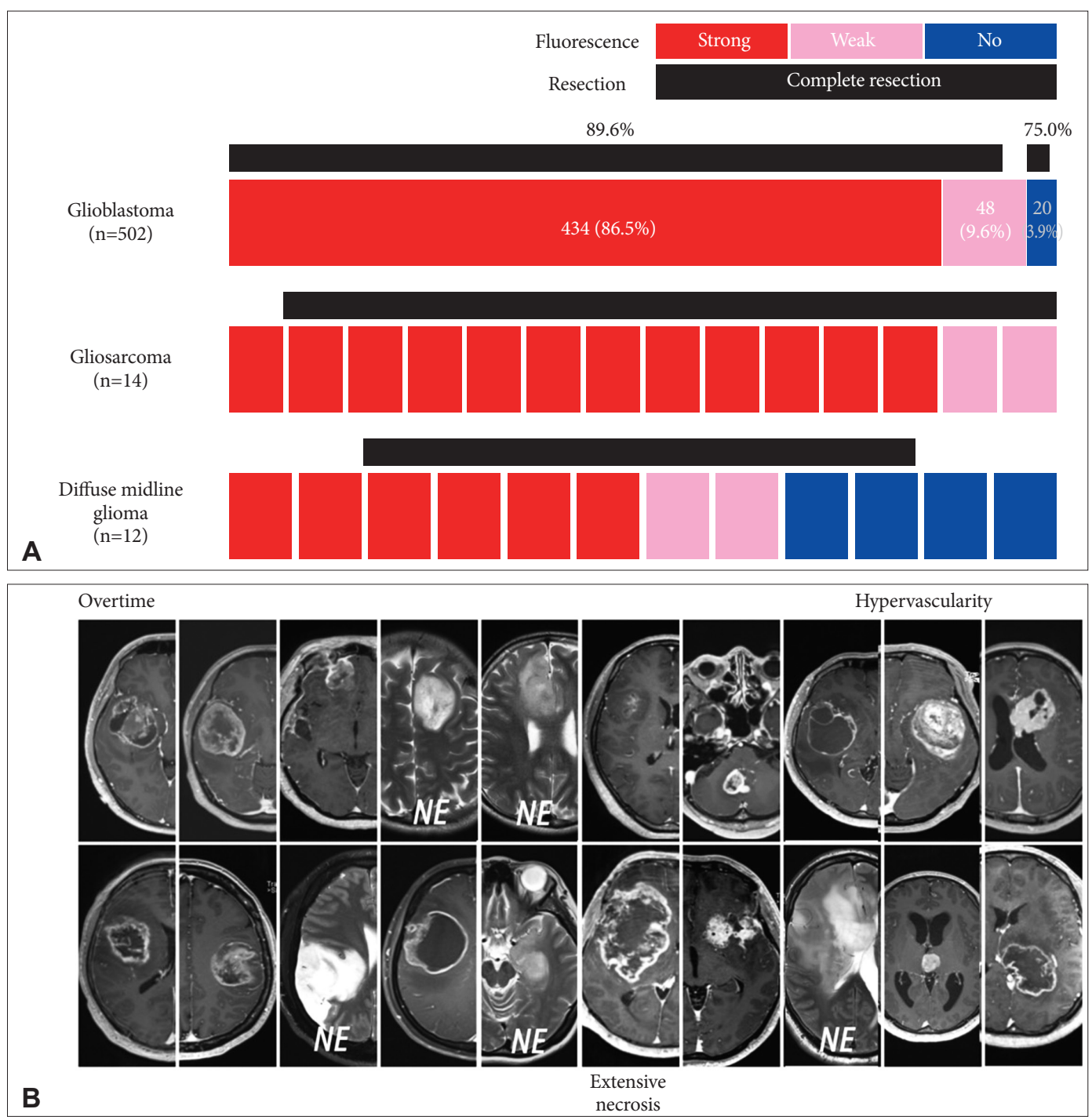

Fig. 2. A: Distribution of fluorescence intensity and complete resection rate of World Health Organization (WHO) grade IV gliomas. Numbers in glioblastoma indicate number of cases, while a single cubic unit in gliosarcoma and diffuse midline glioma denotes individual case. B: Radiological characteristics of 20 glioblastoma cases which showed no fluorescence. Among them, 5 cases showed no enhancement (NE) and represented with T2-weighted MRIs, and the other 15 cases showed strong enhancement in T1-weighted MRIs.

We also experienced each of case of a gliomatosis cerebri and a glioneuronal tumor which were correspondent to WHO grade III with weak fluorescence.

\section{Grade II gliomas}

Of the 87 grade II gliomas, oligodendrogliomas $(57.5 \%$, $\mathrm{n}=50$ ) accounted for the majority (Fig. 4). This is because many oligodendrogliomas have enhanced or high blood volume areas that may be interpreted as malignant lesions in preoperative MRI, which leads to the determination of FGS. As expected, most oligodendrogliomas showed no fluorescence $(82.0 \%)$. However, there were 9 fluorescence positive cases (18.0\%) including 2 strong intensity cases (4.0\%). Ironically, complete resection rate was significantly superior in fluorescence-negative group (92.7\%) to fluorescence-positive group $(66.7 \%, p=0.03)$. The incompletely resected oligodendrogliomas were mainly extensive tumors.

A total of 20 cases of astrocytomas harboring diffuse astrocytomas or oligoastrocytomas were included in this series (Fig. 4). All other 18 cases showed no fluorescence, except for 2 cases with focal fluorescence. Complete resection could be achieved in 15 cases including those fluorescence positive cases. The reasons for incomplete resection in 4 fluorescence negative cases were extensive nature in 3 cases and eloquent area involvement in 2 cases.

We have experienced 9 cases of neurocytomas including 3 cases of central neurocytoma and 6 cases of extraventricular neurocytoma (Fig. 4). Only one of each case of central neurocytoma and extraventricular neurocytoma exhibited weak and strong fluorescence respectively. Unexpectedly, all 7 cases of 


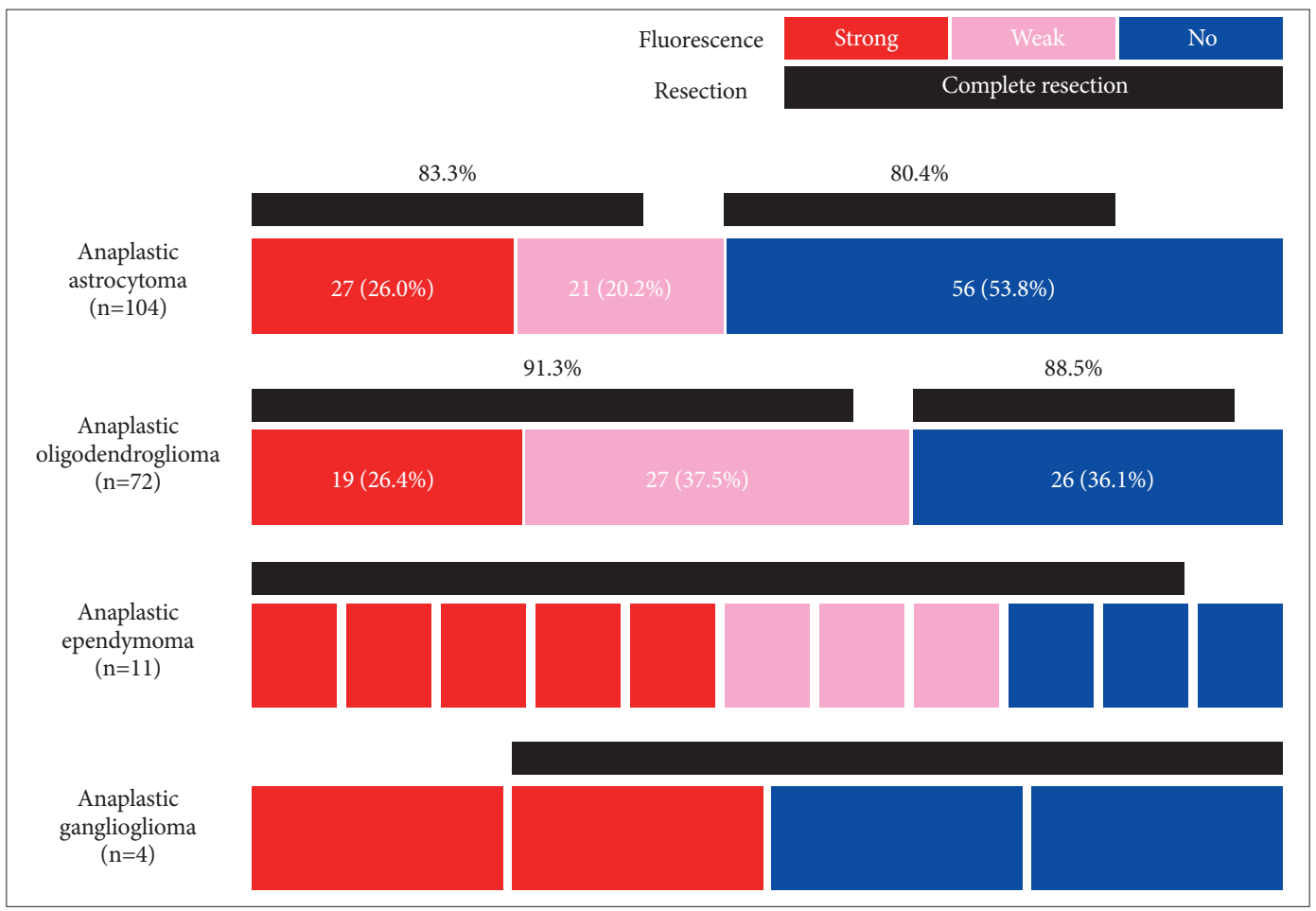

Fig. 3. Distribution of fluorescence intensity and complete resection rate of World Health Organization (WHO) grade III gliomas. Numbers in anaplastic astrocytoma and anaplastic oligodendroglioma indicate number of cases, while a single cubic unit in anaplastic ependymoma and anaplastic ganglioglioma denotes individual case.

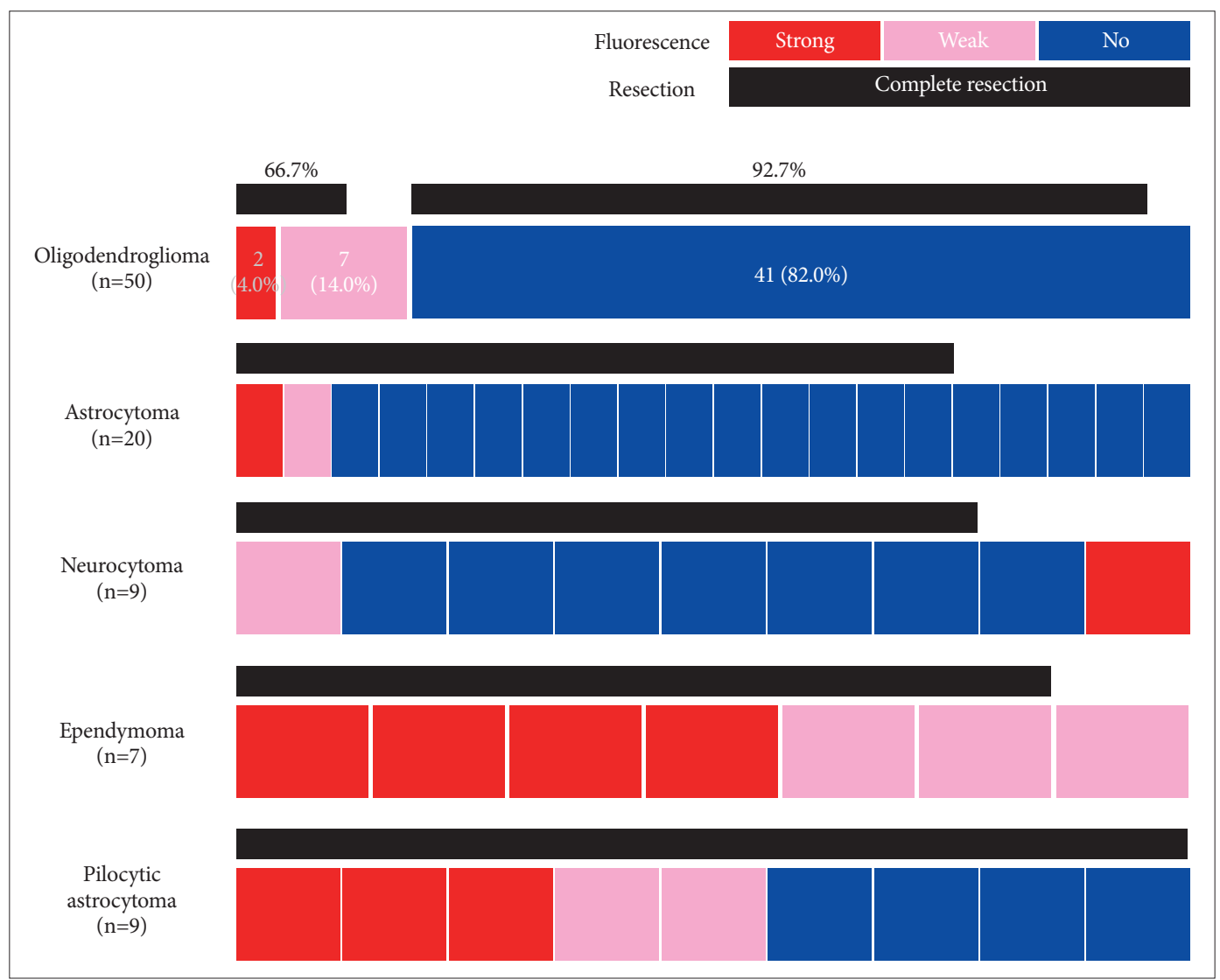

Fig. 4. Distribution of fluorescence intensity and complete resection rate of World Health Organization (WHO) grade II and I gliomas. Numbers in oligodendroglioma indicate number of cases, while a single cubic unit in other tumors denotes individual case. 
ependymomas in this series showed positive fluorescence showing strong intensity in 4 cases and weak intensity in 3 cases (Fig. 4). We also experienced a case of pleomorphic xanthoastrocytoma which showed strong fluorescence positivity.

\section{Grade I glioma}

Notably, WHO grade I gliomas also showed fluorescence in 5 out of 9 pilocytic astrocytomas including 3 strong and 2 weak intensities (Fig. 4). All the pilocytic astrocytomas could be resected completely regardless to fluorescence positivity. However, other WHO grade I gliomas such as gangliogliomas (5 cases), dysembryoplastic neuroepithelial tumors (3 cases), choroid plexus papilloma ( 1 case), and papillary glioneuronal tumor (1 case) showed no fluorescence.

\section{DISCUSSION}

In this study we used the 2016 WHO Classification of Tumors of the Central Nervous System for the diagnosis and included not only gliomas but also minor number of nonpure glioma pathologies such as neuronal and neuronal-glial tumors and choroid plexus tumors. To simplify the classification, subheadings of the genetic signature or NOS are omitted. We left the diagnosis of Glioneuronal tumor grade III, which is not a part of official WHO classification, due to its ambiguity of histology and genetic signatures.

5-ALA induced fluorescence provides the neurosurgeon with real-time information that can distinguish malignant tissue from normal tissue during surgery. Furthermore, FGS allows more extensive resection than neural navigationguided surgery, as 5-ALA-induced fluorescence appears to the tumor infiltration area [19-21]. As a result, it leads to better results by improving the complete resection rate and overall patient survival in glioblastoma [22-24]. Our data also demonstrated the significantly higher complete resection rate in FGS with fluorescence compared with than those without fluorescence in glioblastomas.

Unlike glioblastoma, there were variable results about fluorescence rate in WHO grade III gliomas. Especially, fluorescence rate was substantially different between anaplastic astrocytoma and anaplastic oligodendroglioma despite the same WHO grade (46.2\% in anaplastic astrocytoma and $63.9 \%$ in anaplastic oligodendroglioma). Our previous study suggested that 5-ALA-induced fluorescence is associated with $\mathrm{IDH} 1 \mathrm{mu}-$ tation in WHO grade III gliomas [25]. Since heme synthesis pathway of mutant IDH1 glioma lags behind wild-type IDH1 glioma, mutant IDH1 glioma has stronger fluorescence than wild-type IDH1 glioma due to slowness of the 5-ALA metabolic pathway $[25,26]$. It is true that there are more mutant IDH1 type in anaplastic oligodendroglioma than anaplastic astrocyto- mas. Thus, it is presumed that the incidence of IDH mutational status might have affected the positive fluorescence rate.

Intraoperative fluorescence is affected by several other factors. Fluorescence does not appear in necrotic tissue. Therefore, as is common with glioblastoma, if the tumor has extensive necrosis, it is not fluorescent. Hypervascularity in high-grade gliomas also makes it difficult to detect fluorescence during surgery because of hemorrhage. Moreover, the fluorescence is influenced by extra-tumor factors. It is known that fluorescence intensity is maximum after 4 to 9 hours of oral administration of 5-ALA, and then it gradually reduces for 20 hours [27,28]. In our experience, there was one glioblastoma case which failed to visualize of fluorescence due to failure of adjusting surgical time window. Also, there was a case that had excessive body weight that did not show visible fluorescence, due to the insufficient dose of 5-ALA [29].

Unlike high-grade gliomas, 5-ALA-induced fluorescence plays a lesser role in the removal of low-grade gliomas. Recently, using a fiber-optic probe to measure PpIX, a solution has been reported that can identify low-grade gliomas that do not fluoresce during surgery $[21,30,31]$. However, under current standard microscopes, only 10-20\% low grade gliomas showed visible fluorescence $[17,32,33]$. The papers reported sporadically the positivity of fluorescence in some low grade gliomas, including ependymoma, oligodendroglioma, and pleomorphic xanthoastrocytoma [34,35]. In our experience, pilocytic astrocytoma of WHO grade I showed high rate of positive fluorescence (5 out of 9 cases). And we could observe fluorescence positivity in WHO grade II gliomas including pleomorphic xanthoastrocytoma, ependymoma, oligodendroglioma, extra ventricular neurocytoma, and diffuse astrocytoma. The clinical significance of these fluorescence-positive low-grade gliomas should be further evaluated. The fluorescence in tumor cells have a correlation with an increased proliferation rate and anaplasia parameters $[21,33,36,37]$. In addition, patients with low grade glioma fluorescence have been reported to have shorter overall survival and shorter periods of malignant transformation than patients without fluorescence [38]. Although the fluorescence induced by 5-ALA in low-grade glioma does not have a significant effect on the complete resection as in high grade glioma, the fluorescent area in low-grade glioma are helpful to get tissues for accurate diagnosis and to avoid histological downgrading.

In conclusion, this broad epidemiological data of 5-ALA fluorescence in various grades of glioma provides a basic reference to the clinical application of FGS using 5-ALA in glioma surgery. And FGS's experience with rare gliomas will extend future knowledge and research insights.

\section{Conflicts of Interest}

The authors have no potential conflicts of interest. 


\section{Acknowledgments}

This research was supported by the Bio \& Medical Technology Development Program of the National Research Foundation (NRF) funded by the Ministry of Science \& ICT (NRF-2018M3A9H3021707) in Korea.

\section{REFERENCES}

1. McGirt MJ, Chaichana KL, Attenello FJ, et al. Extent of surgical resection is independently associated with survival in patients with hemispheric infiltrating low-grade gliomas. Neurosurgery 2008;63:700-7; author reply 707-8.

2. Smith JS, Chang EF, Lamborn KR, et al. Role of extent of resection in the long-term outcome of low-grade hemispheric gliomas. J Clin Oncol 2008;26:1338-45

3. Stummer W, Kamp MA. The importance of surgical resection in malignant glioma. Curr Opin Neurol 2009;22:645-9.

4. Stupp R, Hegi ME, Mason WP, et al. Effects of radiotherapy with concomitant and adjuvant temozolomide versus radiotherapy alone on survival in glioblastoma in a randomised phase III study: 5-year analysis of the EORTC-NCIC trial. Lancet Oncol 2009;10:459-66.

5. Brown PD, Maurer MJ, Rummans TA, et al. A prospective study of quality of life in adults with newly diagnosed high-grade gliomas: the impact of the extent of resection on quality of life and survival. Neurosurgery 2005;57:495-504.

6. Stepp H, Stummer W. 5-ALA in the management of malignant glioma. Lasers Surg Med 2018;50:399-419.

7. Teng L, Nakada M, Zhao SG, et al. Silencing of ferrochelatase enhances 5-aminolevulinic acid-based fluorescence and photodynamic therapy efficacy. Br J Cancer 2011;104:798-807.

8. Wolburg H, Noell S, Fallier-Becker P, Mack AF, Wolburg-Buchholz K. The disturbed blood-brain barrier in human glioblastoma. Mol Aspects Med 2012;33:579-89.

9. Zhao SG, Chen XF, Wang LG, et al. Increased expression of ABCB6 enhances protoporphyrin IX accumulation and photodynamic effect in human glioma. Ann Surg Oncol 2013;20:4379-88.

10. Ferraro N, Barbarite E, Albert TR, et al. The role of 5-aminolevulinic acid in brain tumor surgery: a systematic review. Neurosurg Rev 2016;39:545-55.

11. Stummer W, Stocker S, Novotny A, et al. In vitro and in vivo porphyrin accumulation by $\mathrm{C} 6$ glioma cells after exposure to 5-aminolevulinic acid. J Photochem Photobiol B 1998;45:160-9.

12. Hervey-Jumper SL, Berger MS. Maximizing safe resection of low- and high-grade glioma. J Neurooncol 2016;130:269-82.

13. Kim SK, Choi SH, Kim YH, Park CK. Impact of fluorescence-guided surgery on the improvement of clinical outcomes in glioblastoma patients. Neurooncol Pract 2014;1:81-5.

14. Roberts DW, Valdés PA, Harris BT, et al. Glioblastoma multiforme treatment with clinical trials for surgical resection (aminolevulinic acid). Neurosurg Clin N Am 2012;23:371-7.

15. Stummer W, Tonn JC, Mehdorn HM, et al. Counterbalancing risks and gains from extended resections in malignant glioma surgery: a supplemental analysis from the randomized 5 -aminolevulinic acid glioma resection study. Clinical article. J Neurosurg 2011;114:613-23.

16. Zhang C, Boop FA, Ruge J. The use of 5-aminolevulinic acid in resection of pediatric brain tumors: a critical review. J Neurooncol 2019;141:567-73.

17. Jaber M, Wölfer J, Ewelt C, et al. The value of 5-aminolevulinic acid in low-grade gliomas and high-grade gliomas lacking glioblastoma imaging features: an analysis based on fluorescence, magnetic resonance imaging, 18F-fluoroethyl tyrosine positron emission tomography, and tumor molecular factors. Neurosurgery 2016;78:401-11; discussion 411.

18. Kim S, Kim JE, Kim YH, et al. Glutaminase 2 expression is associated with regional heterogeneity of 5 -aminolevulinic acid fluorescence in glioblastoma. Sci Rep 2017;7:12221.

19. Roessler K, Becherer A, Donat M, Cejna M, Zachenhofer I. Intraoperative tissue fluorescence using 5-aminolevolinic acid (5-ALA) is more sen- sitive than contrast MRI or amino acid positron emission tomography ((18)F-FET PET) in glioblastoma surgery. Neurol Res 2012;34:314-7.

20. Schucht P, Knittel S, Slotboom J, et al. 5-ALA complete resections go beyond MR contrast enhancement: shift corrected volumetric analysis of the extent of resection in surgery for glioblastoma. Acta Neurochir (Wien) 2014;156:305-12; discussion 312.

21. Hadjipanayis CG, Widhalm G, Stummer W. What is the surgical benefit of utilizing 5 -aminolevulinic acid for fluorescence-guided surgery of malignant gliomas? Neurosurgery 2015;77:663-73.

22. Marko NF, Weil RJ, Schroeder JL, Lang FF, Suki D, Sawaya RE. Extent of resection of glioblastoma revisited: personalized survival modeling facilitates more accurate survival prediction and supports a maximumsafe-resection approach to surgery. J Clin Oncol 2014;32:774-82.

23. McGirt MJ, Chaichana KL, Gathinji M, et al. Independent association of extent of resection with survival in patients with malignant brain astrocytoma. J Neurosurg 2009;110:156-62.

24. Díez Valle R, Hadjipanayis CG, Stummer W. Established and emerging uses of 5-ALA in the brain: an overview. J Neurooncol 2019;141:487-94.

25. Kim JE, Cho HR, Xu WJ, et al. Mechanism for enhanced 5-aminolevulinic acid fluorescence in isocitrate dehydrogenase 1 mutant malignant gliomas. Oncotarget 2015;6:20266-77.

26. Saito K, Hirai T, Takeshima H, et al. Genetic factors affecting intraoperative 5-aminolevulinic acid-induced fluorescence of diffuse gliomas. Radiol Oncol 2017;51:142-50.

27. Haj-Hosseini N, Richter JC, Hallbeck M, Wårdell K. Low dose 5-aminolevulinic acid: implications in spectroscopic measurements during brain tumor surgery. Photodiagnosis Photodyn Ther 2015;12:209-14.

28. Chung IW, Eljamel S. Risk factors for developing oral 5-aminolevulinic acid-induced side effects in patients undergoing fluorescence guided resection. Photodiagnosis Photodyn Ther 2013;10:362-7.

29. GmbH M. Gliolan(R) product information. Wedel: Medac, 2014. (Accessed September 23, 2015, at http://www.ema.europa.eu/docs/en_GB/ document_library/EPAR_-_Product_Information/human/000744/ WC500021790.pdf).

30. Valdés PA, Leblond F, Kim A, et al. Quantitative fluorescence in intracranial tumor: implications for ALA-induced PpIX as an intraoperative biomarker. J Neurosurg 2011;115:11-7.

31. Valdés PA, Kim A, Leblond F, et al. Combined fluorescence and reflectance spectroscopy for in vivo quantification of cancer biomarkers in low- and high-grade glioma surgery. J Biomed Opt 2011;16:116007.

32. Nishikawa R. Fluorescence illuminates the way.... Neuro Oncol 2011;13:805.

33. Widhalm G, Kiesel B, Woehrer A, et al. 5-Aminolevulinic acid induced fluorescence is a powerful intraoperative marker for precise histopathological grading of gliomas with non-significant contrast-enhancement. PLoS One 2013;8:e76988.

34. Marbacher S, Klinger E, Schwyzer L, et al. Use of fluorescence to guide resection or biopsy of primary brain tumors and brain metastases. Neurosurg Focus 2014;36:E10.

35. Schwake M, Schipmann S, Müther M, Köchling M, Brentrup A, Stummer W. 5-ALA fluorescence-guided surgery in pediatric brain tumorsa systematic review. Acta Neurochir (Wien) 2019;161:1099-108.

36. Ewelt C, Floeth FW, Felsberg J, et al. Finding the anaplastic focus in diffuse gliomas: the value of Gd-DTPA enhanced MRI, FET-PET, and intraoperative, ALA-derived tissue fluorescence. Clin Neurol Neurosurg 2011;113:541-7.

37. Widhalm G, Wolfsberger S, Minchev G, et al. 5-Aminolevulinic acid is a promising marker for detection of anaplastic foci in diffusely infiltrating gliomas with nonsignificant contrast enhancement. Cancer 2010;116:1545-52.

38. Jaber $\mathrm{M}$, Ewelt $\mathrm{C}$, Wölfer J, et al. Is visible aminolevulinic acid-induced fluorescence an independent biomarker for prognosis in histologically confirmed (World Health Organization 2016) low-grade gliomas? Neurosurgery 2019;84:1214-24. 